\title{
Performance of the modified TRISS for evaluating trauma care in subpopulations: A cohort study
}

\author{
Leonie de Munter ${ }^{\mathrm{a}, *}$, Suzanne Polinder ${ }^{\mathrm{b}}$, Daan Nieboer ${ }^{\mathrm{b}}$, Koen W.W. Lansink ${ }^{\mathrm{a}, \mathrm{c}, \mathrm{d}}$, \\ Ewout W. Steyerberg ${ }^{\mathrm{b}, \mathrm{e}}$, Mariska A.C. de Jongh ${ }^{\mathrm{a}, \mathrm{c}}$
}

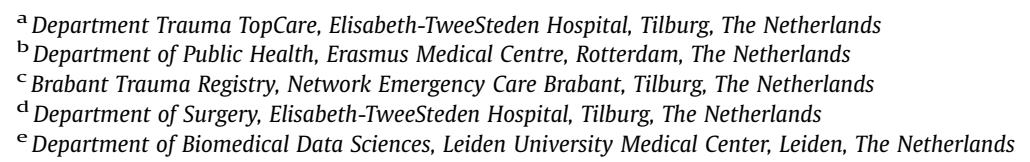

A RTICLE IN F O

\section{Article history:}

Accepted 29 March 2018

\section{Keywords:}

Prediction model

Performance

TRISS

Trauma

Subpopulations

\begin{abstract}
A B S T R A C T
Introduction: Previous research showed that there is no agreement on a practically applicable model to use in the evaluation of trauma care. A modification of the Trauma and Injury Severity Score (modified TRISS) is used to evaluate trauma care in the Netherlands. The aim of this study was to evaluate the prognostic ability of the modified TRISS and to determine where this model needs improvement for better survival predictions.

Methods: Patients were included if they were registered in the Brabant Trauma Registry from 2010 through 2015. Missing values were imputed according to multiple imputation. Subsets were created based on age, length of stay, type of injury and injury severity. Probability of survival was calculated with the modified TRISS. Discrimination was assessed with the Area Under the Receiver Operating Curve (AUROC). Calibration was studied graphically.

Results: The AUROC was $0.84(95 \% \mathrm{CI}: 0.83,0.85)$ for the total cohort $(\mathrm{N}=69747)$ but only $0.53(95 \% \mathrm{CI}$ : $0.51,0.56$ ) for elderly patients with hip fracture. Overall, calibration of the modified TRISS was adequate for the total cohort, with an overestimation for elderly patients and an underestimation for patients without brain injury.

Conclusions: Outcome comparison conducted with TRISS-based predictions should be interpreted with care. If possible, future research should develop a simple prediction model that has accurate survival prediction in the aging overall trauma population (preferable with patients with hip fracture), with readily available predictors.
\end{abstract}

(c) 2018 Elsevier Ltd. All rights reserved.

\section{Introduction}

Prediction models that adequately predict survival are required to determine the quality of care in trauma patients. Trauma is a major cause of mortality in young adults worldwide [1]. In 2014, almost 84000 patients were admitted due to injuries in the Netherlands and the 30-day mortality rate was 2.1\% [2]. Scoring systems and prediction models are important tools to quantify the

\footnotetext{
* Corresponding author at: Elisabeth-TweeSteden Hospital, Department Trauma TopCare, Hilvarenbeekseweg 60, 5000 LC Tilburg, The Netherlands.

E-mail addresses: l.demunter@etz.nl (L. de Munter), s.polinder@erasmusmc.nl (S. Polinder),d.nieboer@erasmusmc.nl (D. Nieboer), k.lansink@etz.nl

(K.W.W. Lansink), e.steyerberg@erasmusmc.nl (E.W. Steyerberg), m.d.jongh@nazb.nl (M.A.C. de Jongh).
}

probability of survival and to evaluate and improve the quality of care for the large number of injured patients [3].

The Trauma and Injury Severity Score (TRISS) was developed from the Major Trauma Outcome Study (MTOS) in 1987 to evaluate the quality of trauma care by comparing outcomes with a norm score [4]. The TRISS is a weighted score based on the Injury Severity Score (ISS), age, and the coded Revised Trauma score (RTS). The RTS combines the Glasgow Coma Score (GCS), Systolic Blood Pressure (SBP) and Respiratory Rate (RR). The MTOS was a retrospective study conducted in North America from 1982 through 1987 and was of great value for the development of TRISS. It has been shown previously that the TRISS has several limitations [5,6]. The use of TRISS in an external population raises concerns, because differences between cohorts are distinct [7,8].

Previous research in the Dutch population demonstrated an adequate performance of the TRISS with coefficients from the 
MTOS or from the National Trauma Data Bank (NTDB) in the total population, but demonstrated a poor reflection of the mortality risk of elderly patients (with hip fractures) [9-12]. Furthermore, Frankema et al. [13] suggested developing and using a more accurate model for the evaluation of trauma care in the Dutch trauma population. A recent review showed that there is no agreement on a better and practically applicable model to use in the evaluation of trauma care [14].

In 2015 the Dutch Trauma Registry developed a new model based solely on the variables in the TRISS model according to their trauma population, including the elderly patients with hip fracture [15]. This model is used to compare quality of care between Dutch hospitals, but has never been validated in subsets. The aim of this study was to determine the performance of the modified TRISS in subpopulations and to determine where this model needs improvement for better survival predictions in the Dutch trauma population.

\section{Methods}

\section{Study population and data collection}

At present, the Netherlands consists of eleven trauma regions, all including a coordinating trauma level I center. The region Noord-Brabant is representative of the total Dutch trauma population. It covers $16 \%$ of all admitted trauma patients in the Netherlands and includes urban as well as rural populations [2]. Eleven hospitals in the region Noord-Brabant contributed to the Brabant Trauma Registry (BTR), including one level I hospital and ten level II or III hospitals. The registry database contains data of all trauma patients in Noord-Brabant that were admitted after visiting the Emergency Department (ED) within $48 \mathrm{~h}$ after a trauma, independent of injury severity or injury type. Secondary referrals and patients who die in the ED were also registered. Patients who were dead on arrival were excluded from the registry.

A total of 72411 patients were registered in the BTR from 2010 through 2015. Variables collected in this registry included demographics, SBP, RR, GCS, ISS, trauma mechanism (blunt vs. penetrating) and Abbreviated Injury Scale (AIS)-codes (version 1998) [16]. In-hospital mortality was considered as the primary outcome measure.

\section{Model}

The Probability of survival (Ps) was calculated using the natural logarithm (Logit) of the modified TRISS:

Logit $(\mathrm{Ps})=\alpha_{\mathrm{i}}+\beta_{\mathrm{GCS}, \mathrm{i}}{ }^{*} \mathrm{GCS}+\beta_{\mathrm{RR}, \mathrm{i}}{ }^{*} \mathrm{RR}+\beta_{\mathrm{SBP}, \mathrm{i}}{ }^{*} \mathrm{SBP}+\beta_{\mathrm{ISS}, \mathrm{i}}{ }^{*} \mathrm{ISS}+\beta_{\mathrm{age}}$ i*age

Age was equal to 0 if the patient was $<55$ years and equal to 1 if the patient was $\geq 55$ years of age. The coefficients were derived from the Dutch Trauma Registry in 2015 (Table 1) [15].

Table 1

The estimated coefficients $\left(b_{n}\right)$ of the modified TRISS as used in this study.

\begin{tabular}{ll}
\hline & Coefficients $^{\mathrm{a}}$ \\
Mechanism of Injury & Blunt \\
\hline Intercept & 1.5090 \\
RR & 0.2372 \\
SBP & 0.6460 \\
GCS & 0.4008 \\
ISS & -0.1087 \\
Age & -2.2091 \\
\hline
\end{tabular}

List of abbreviations: GCS, Glasgow Coma Scale; ISS, Injury Severity Score; RR, Respiratory Rate; SBP, Systolic Blood Pressure.

a All coefficients are for blunt injuries. Penetrating injuries have different coefficients (data not shown).

\section{Subsets}

Analyses were performed on the total cohort (with and without elderly patients with hip fractures) and on several subsets. Elderly patients with hip fractures (patients $\geq 65$ years and an ISS $\leq 13$, suffering an isolated fracture of the proximal femur [defined as: AIS 1998 codes 851808.3, 851810.3 and 851812.3]) were excluded in the subsets, because it was previously suggested that those patients should be excluded from prediction modeling [17,18]. Each subset represents different challenges to the trauma centers, and were based on:

- Age:

- Elderly, including only patients $>75$ years

- Children, including only patients $\leq 15$ years

- Type of injury: Traumatic Brain Injury (TBI), defined as AIShead $\geq 3$

- Injury severity: Major trauma, defined as ISS $>15$

- LOS $>2$, including patients who die during the first 2 days of admission.

- Trauma center (including only patients admitted to a trauma center level I) and non-trauma center (including patients admitted to a trauma center level II or III).

\section{Statistical analysis}

Prehospital coded values for the V component of GCS and RR were selected in patients that were sedated and/or intubated, instead of the registered hospital values. Also, prehospital values for the E and M component of GCS were selected in patients that were sedated. Data were screened for missing values. ISS, RTS and age for patients with missing outcome values and with unknown mechanism of injury were compared to patients with known outcome values. Missing value patterns were analyzed for GCS, SBP, RR and ISS. The components of the GCS were transformed into dummy variables and RR was log-transformed in the imputation process. We assumed that missing values were Missing At Random (MAR) [19] and imputed missing values using the following variables: mortality, mechanism of injury, ISS, eye/motor/verbal (E, $\mathrm{M}$ and $\mathrm{V}$ ) component of GCS, SBP, RR, age, with 45 imputations and 10 iterations. Sensitivity analysis was performed in which only complete cases were included.

The performance measures that were used in this study were discrimination and calibration. Discrimination was calculated using the Area Under the Receiver Operating Curve (AUROC), including a 95\% confidence interval (95\% CI). Differences between AUROC were considered significant if the 95\% CI did not overlap. Calibration was assessed graphically using calibration plots. In calibration plots the agreement between observed proportion of survival and the predicted probability is visualized using restricted cubic splines.

The performance of modified TRISS for the total group of patients was compared with the specific subsets, to determine the effect of inclusion and exclusion of the subsets. The statistical programs IBM SPSS version 24 (Chicago, USA) and R version 3.4.0 ( $R$ Foundation for Statistical Computing, Vienna, Austria) were used for the analyses.

\section{Results}

Baseline characteristics

A total of 72411 patients were included in the BTR. Patients with penetrating injury $(\mathrm{N}=2539)$ were excluded because the number of non-survivors $(\mathrm{N}=32)$ was too low to interpret the results of 
discrimination and calibration. Patients with unknown survival outcome $(\mathrm{N}=125)$ were excluded from analysis. The excluded patients did not differ in age, RTS, or ISS from the total cohort (data not shown).

There were 69747 patients used for further analysis, including 11514 elderly patients with hip fracture. The eye component, motor component and verbal component of the Glasgow Coma Scale were missing in 5995 (8.6\%), 6024 (8.6\%) and 6162 (8.8\%) observations, respectively. SBP was missing in 11777 observations (16.9\%), RR in 30083 observations (43.1\%) and ISS was missing in 158 patients $(0.2 \%)$.

The mortality rate decreased $(1.7 \%$ vs. $2.1 \%)$ in the total cohort after exclusion of elderly patients with hip fractures (Table 2 ). Next, the exclusion of children from the BTR resulted in an increase in mortality rate $(2.1 \%)$. However, the ISS (median [IQR]) remained the same. Excluding the elderly from the BTR resulted in a higher percentage of men (60.7\%) and a lower mortality rate $(0.9 \%)$, compared to the total cohort.

The exclusion of non-TBI patients and minor injury resulted in older age (55.3 (SD: 23.9) and 60.6 (SD: 22.3) respectively) and higher mortality rates ( $12.1 \%$ and $12.8 \%$ respectively). Patients with LOS $>2$ had a mean (SD) age of 60.6 (22.9) and a mortality rate of $3.4 \%$.

Patients in the level I trauma center were younger (45.4 [SD: 27.6]), had a higher mortality rate (4.5\%), and had a higher median ISS (9 [IQR: 4-11]) compared to the level II and III trauma centers (mortality rate: $1.2 \%$, age: 47.9 [SD:27.9] and ISS [IQR]: 5 [4-9]).

\section{Discrimination}

No differences were found between complete case analysis and the imputed dataset for discrimination (data not shown). The AUROC of the total cohort was $0.84(95 \% \mathrm{CI}: 0.83,0.85)$ and for the elderly patients with hip fracture 0.53 (95\% CI: 0.51, 0.56) (Fig. 1). After exclusion of the elderly patients with hip fracture the AUROC of the different subsets ranged from $0.72(95 \% \mathrm{CI}: 0.70,0.74)$ for the subset older than 75 years to 0.93 (95\% CI: $0.91,0.94)$ for the subset level I trauma center. The subset with exclusion of children, the subset with elderly patients, the selections based on ISS, LOS $>2$ days and the subset with only level II and III trauma centers decreased the discriminative ability significantly. Discrimination was significantly higher in the Level I trauma center than in the level II and III trauma centers.

\section{Calibration}

Calibration curves for the modified TRISS were shown in Fig. 2. There were no apparent differences between calibration of the total cohort and the complete cases. The total cohort showed predictions close to the identity line, thus equal observed proportion and predicted probabilities. After exclusion of the elderly patients with hip fracture, the cohort showed higher observed proportion of survival compared to the predicted probabilities in the highest Ps bins (0.8-1.0).

The subset $\leq 75$ years old showed a higher observed proportion of survival compared to the total cohort, especially in the highest Ps bins (0.5-1.0). The subset of elderly showed a significant overestimation of the predicted survival rate.

The calibration curve of the TBI subset showed an underestimation of the predicted probabilities of the TRISS in the lower Ps bins (0.0-0.3) and an overestimation of the higher Ps bins (0.3-1.0). The non-TBI patients showed a significant underestimation of the predicted probability of survival in all probability bins. Calibration of the subset with an ISS $>15$ showed a similar curve as the TBI subset. The subset LOS $>2$ showed an calibration curve close to the identity line. The level I trauma center had higher observed proportion survivors in the lower Ps bins (0.0-0.7) but lower observed proportion in the higher Ps bins (0.7-1.0) compared to the predicted probabilities. The calibration curve of the subset with only level II and level III trauma centers was close to the identity line.

\section{Discussion}

Prediction models need to be reliable if used in evaluating the quality of trauma care. Although discrimination of the modified TRISS in the total trauma cohort was adequate, the model performed much better when excluding elderly, with or without hip fractures. Overall, calibration of the modified TRISS was adequate for the total cohort. However, the model overestimates the survival for the elderly and underestimates survival for patients without TBI.

Discrimination of a model is dependent on the distribution of prognostic factors. Discrimination in the elderly (with hip fractures) could be low because the heterogeneity of the case-mix decreased. In contrast to discrimination, calibration of the cohort excluding elderly with hip fractures showed no differences compared to calibration of the total cohort. The lack of differences in the calibration plot could be explained by the high number of patients remaining in the highest predicted probability group after excluding the relative low number of elderly with hip fractures. The NTDB encourages researchers to use inclusion and exclusion criteria in the data registry to create a more homogeneous group; for example, hip fractures should be excluded or analyzed separately, which is also confirmed by Gomez et al. [17,18]. In

Table 2

Study characteristics of the Brabant Trauma Registry with patient subsets from 2010 through 2015.

\begin{tabular}{|c|c|c|c|c|c|c|c|c|c|c|}
\hline & \multicolumn{10}{|c|}{ Brabant Trauma Registry } \\
\hline & \multicolumn{2}{|c|}{$\begin{array}{l}\text { Including elderly with hip } \\
\text { fractures }^{\text {a }}\end{array}$} & \multicolumn{8}{|c|}{ Excluding elderly with hip fractures ${ }^{\mathrm{a}}$} \\
\hline & \multirow[t]{2}{*}{ Total cohort } & \multirow[t]{2}{*}{ Hip fracture ${ }^{a}$} & \multirow[t]{2}{*}{ Total cohort } & \multicolumn{2}{|l|}{ Age (years) } & \multicolumn{3}{|c|}{ Trauma severity } & \multicolumn{2}{|c|}{ Trauma center } \\
\hline & & & & $>15$ & $\leq 75$ & $\mathrm{TBI}^{\mathrm{b}}$ & Major trauma $^{c}$ & LOS $>2$ & Level I & Level II and III \\
\hline $\mathrm{N}$ & 69747 & 11514 & 58233 & 47565 & 46150 & 3531 & 3932 & 28883 & 8443 & 49790 \\
\hline Age (mean, SD) & $53.2(28.6)$ & $82.0(7.7)$ & $47.5(27.8)$ & $56.6(22.2)$ & $38.0(23.0)$ & $55.3(23.9)$ & $55.7(22.3)$ & $60.6(22.9)$ & $45.4(27.6)$ & $47.9(27.9)$ \\
\hline Male (\%) & 49.7 & 27.9 & 53.9 & 53.0 & 60.7 & 62.3 & 65.7 & 47.6 & 58.5 & 53.2 \\
\hline In-hospital mortality $(\%, \mathrm{~N})$ & $2.1(1490)$ & $4.4(502)$ & $1.7(988)$ & $2.1(982)$ & $0.9(406)$ & $12.1(428)$ & $12.8(503)$ & $3.4(975)$ & $4.5(381)$ & $1.2(605)$ \\
\hline RTS (mean, SD) & $7.7(0.4)$ & $7.8(0.3)$ & $7.7(0.5)$ & $7.7(0.5)$ & $7.7(0.5)$ & $7.1(1.3)$ & $7.2(1.3)$ & $7.7(0.6)$ & $7.5(0.9)$ & $7.7(0.4)$ \\
\hline ISS (median, IQR) & $9(4-9)$ & $9(9-9)$ & $5(4-9)$ & $5(4-9)$ & $5(4-9)$ & $17(11-25)$ & $20(17-26)$ & $9(4-9)$ & $9(4-11)$ & $5(4-9)$ \\
\hline ISS (mean, SD) & $7.5(5.2)$ & $9.0(0.2)$ & $7.2(5.7)$ & $7.4(6.1)$ & $7.2(5.7)$ & $19.2(9.5)$ & $22.9(8.6)$ & $8.7(6.7)$ & $10.2(9.4)$ & $6.7(4.6)$ \\
\hline
\end{tabular}

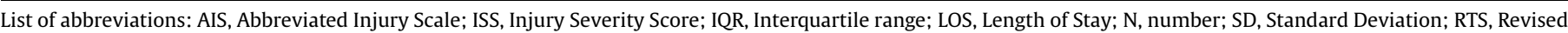
Trauma Score; TBI, Traumatic Brain Injury.

a Patients with hip fracture were considered equal to or older than 65 years, ISS $<13$ and one of the following AIS 1998 codes $851808.3,851810.3$ or 851812.3.

b Patients with TBI were defined as AIS-head $\geq 3$.

c Major trauma was defined as ISS $>15$. 


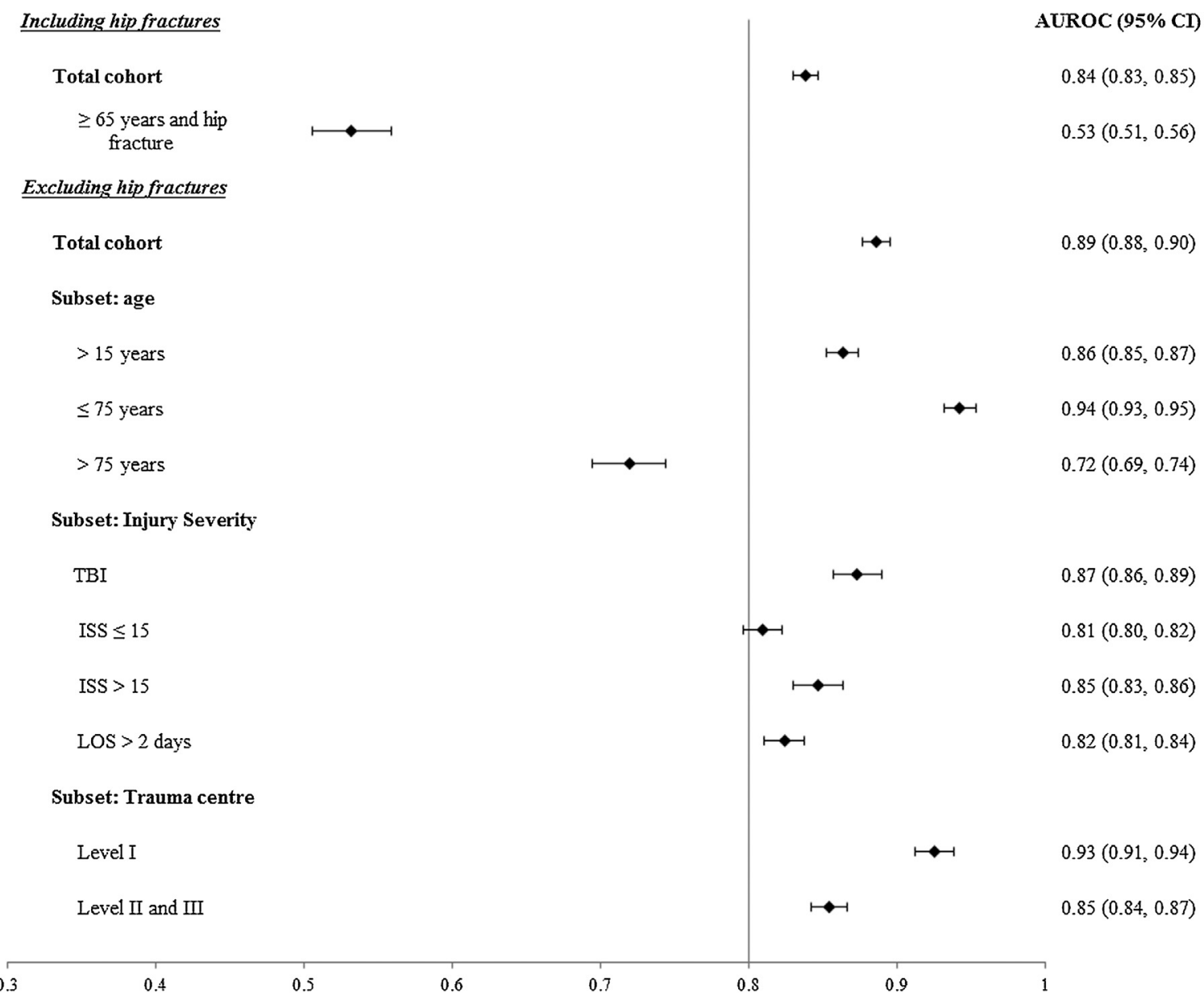

Fig. 1. Discrimination of the modified TRISS for the total cohort and subpopulations.

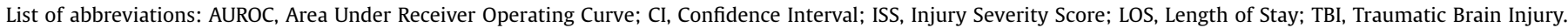

*Patients with hip fracture were considered equal to or older than 65 years, ISS $<13$ and one of the following AIS 1998 codes $851808.3,851810.3$ or 851812.3 .

contrast, others argue that elderly with isolated hip fracture should be included in the trauma registry [20]. Elderly with hip fracture comprise currently $17 \%$ of the total Dutch trauma population [2]. Due to the aging population and the high incidence of falls within these often frail patients, the number of elderly in the trauma registries with hip fractures will increase the following decades. However, this study supports the fact that elderly with hip fracture should be excluded for general trauma center benchmarking when the modified TRISS is used and should be analyzed separately for benchmarking purposes using a more specific prediction model. Nevertheless, if it could be achieved to develop a model with accurate predictions in all subsets, it is preferable to include elderly with hip fractures for evaluation of quality care, to cover all trauma related injuries.

Another explanation of the moderate discrimination in elderly could be explained by the lack of measures for frailty in the model. Next to frailty, dichotomization of age leads to a loss of information, and could be one of the main reasons for the poor performance of the modified TRISS in the elderly [21,22]. Also, elderly often suffer from comorbidities, which could be important predictors of mortality in the aging population [17,23-25]. Some of these issues were already incorporated in previously developed models [26-28]. However, comorbidity measures and frailty are not incorporated in the Dutch Trauma Registry and could therefore not be used in benchmarking trauma care.

The TBI subset had accurate predictions among the lower survival probabilities intervals in the TBI subset. However, the higher intervals showed an overestimation of the survival predictions. Previous research suggested an inability of ISS to account enough for multiple injuries to the same body region [29]. While mortality is often attributed to TBI, severe TBI is often not entirely captured by a measure such as the ISS. Champion et al. [30] suggested to include all anatomic injuries for more accurate survival prediction in patients with TBI.

There are some limitations to this study. First, the modified TRISS is developed in the Dutch trauma population, including the BTR. Results could differ if this model is validated in an external cohort (i.e. a cohort that is not incorporated in the Dutch trauma registry). The study may not be generalizable to other settings with different patient composition. Second, missing values are a common problem in trauma registries. Ignoring them could influence the results and decrease the statistical power. Multiple imputation is an increasingly chosen solution to minimize bias and increase precision [31-33]. The imputation model applied in this study was based on literature [34]. Therefore, we assume that no major bias occurred, as confirmed by the similar results in a complete case analysis. However, it could also be argued to exclude predictors with a high proportion of missing values (e.g. RR in the modified TRISS) for more optimal predictions. Next, patients with unknown mechanism of injury $(\mathrm{N}=1172)$ were included as patients with blunt injuries. It is unlikely this influenced the results, since most trauma patients have blunt injury [2]. In addition, the use of AIS98 is considered a shortcoming. The BTR included AIS08 codes from 2015 onwards, but conversion of AIS98 to AIS08 showed to be unreliable [35]. However, to obtain power to 

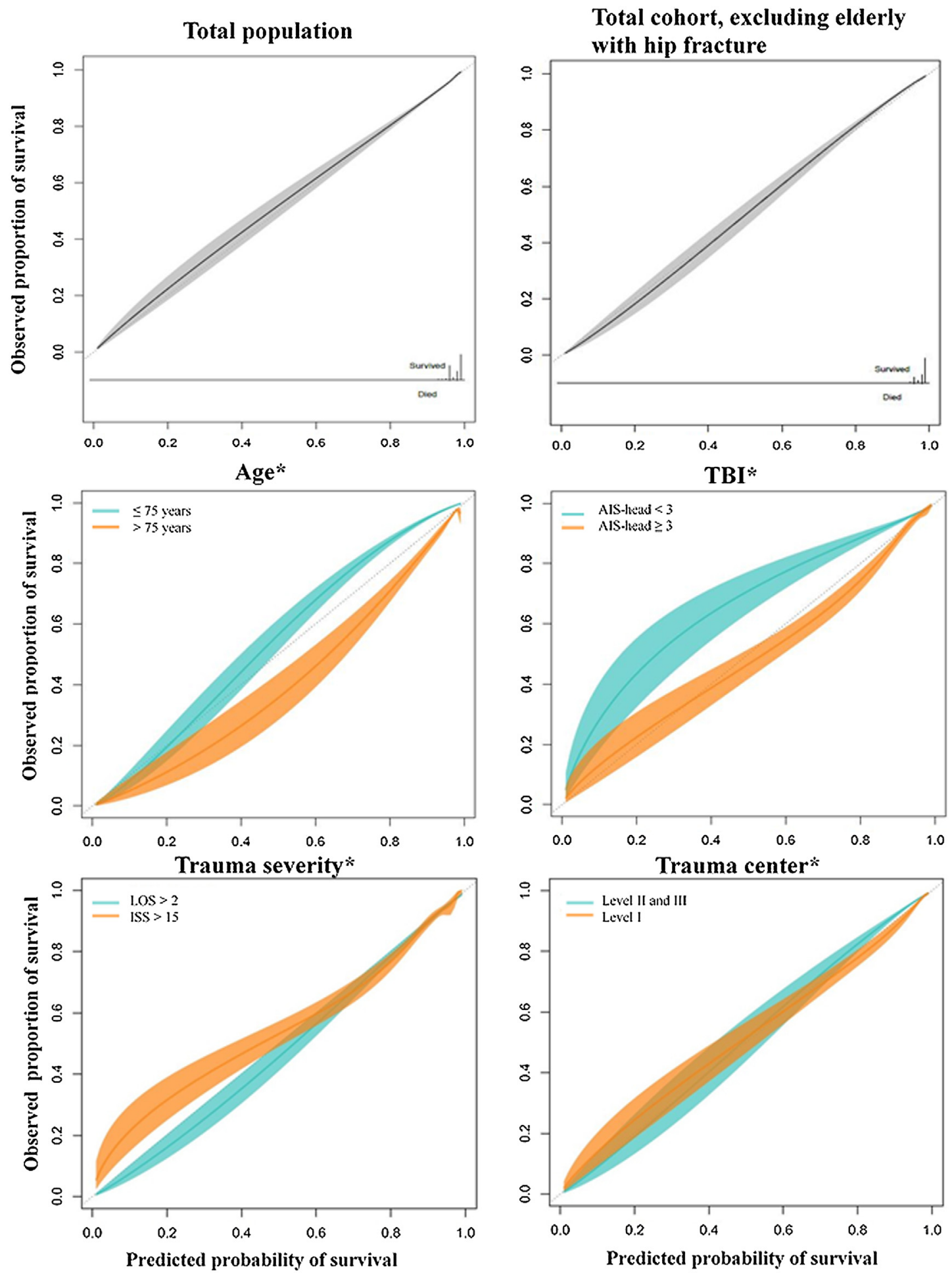

Fig. 2. Calibration curves for the modified TRISS in the total cohort and in different subsets of patients.

List of abbreviations: ISS, Injury Severity Score; LOS, Length of Stay; TBI, Traumatic Brain Injury.

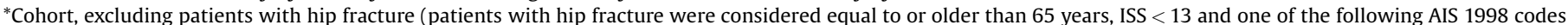
$851808.3,851810.3$ or 851812.3 ).

assess performances among subsets of the registry more years with old AIS98 codes were used, instead of two years with newer AIS08 codes. Last, we note that the outcome measure, in-hospital mortality, is a poor measure of outcome. A better outcome would be 30-day mortality. However, this outcome is not reported in the trauma registry.
The current overall mortality rate in the Netherlands of the acute hospitalized trauma population is only $2 \%$. Therefore, it could be suggested that mortality is not the most important outcome to evaluate trauma care. With the decrease in mortality rates in the developed countries, trauma care could also be assessed with nonfatal outcome measures. 
Outcome comparison conducted with the modified TRISS should be interpreted with care, because the performance of the model is highly dependent on the case mix of the patients included in the registry. The quality of care in the elderly should not be evaluated when the modified TRISS is used. If it could be achieved in the future to develop a model with accurate predictions in all subsets, it is preferable to include elderly for evaluation of quality care to cover all trauma related injuries. Predictors should be readily available and easy to collect in the current trauma registry.

\section{Conflicts of interest}

The authors declare no conflict of interest.

\section{Acknowledgement}

This work was supported by a grant of the Dutch organization for health research and care innovation (ZonMW) section TopCare projects (grantnumber: 80-84200-98-14226). ZonMw had no role in the design, analyses or interpretation of this study.

\section{References}

[1] World Health Organization. Injuries and violence: the facts. 2014 WO700 (August).

[2] Landelijke Netwerk Acute Zorg. Traumazorg in beeld: Landelijke traumaregistratie 2012-2016. rapportage nederland; 2017.

[3] Rutledge R, Osler T, Emery S, Kromhout-Schiro S. The end of the injury severity score (ISS) and the trauma and injury severity score (TRISS): ICISS, an international classification of diseases, ninth revision-based prediction tool, outperforms both ISS and TRISS as predictors of trauma patient survival, hospital charges, and hospital length of stay. J Trauma 1998;44(1):41-9.

[4] Champion HR, Sacco WJ, Hunt TK. Trauma severity scoring to predict mortality. World J Surg 1983;7(1):4-11.

[5] Champion HR, Copes WS, Sacco WJ, Lawnick MM, Keast SL, Frey CF. The major trauma outcome study: establishing national norms for trauma care. J Trauma 1990;30(11):1356-65.

[6] Rogers FB, Osler T, Krasne M, Rogers A, Bradburn EH, Lee JC, et al. Has TRISS become an anachronism? A comparison of mortality between the national trauma data bank and major trauma outcome study databases. J Trauma 2012;73(2):326-31.

[7] Demetriades D, Chan LS, Velmahos G, Berne TV, Cornwell 3rd EE, Belzberg H, et al. TRISS methodology in trauma: the need for alternatives. Br J Surg 1998;85 (3):379-84.

[8] Cayten C, Stahl W, Murphy J, Agarwal N, Byrne D. Limitations of the TRISS method for interhospital comparisons: a multihospital study. J Trauma 1991;31(4):471-82.

[9] Champion H, Sacco W, Copes W. Injury severity scoring again. J Trauma 1995;38(1):94-5.

[10] Schluter PJ, Nathens A, Neal ML, Goble S, Cameron CM, Davey TM, et al. Trauma and injury severity score (TRISS) coefficients 2009 revision. J Trauma 2010;68 (4):761-70.

[11] De Jongh MAC, Verhofstad MHJ, Leenen LPH. Accuracy of different survival prediction models in a trauma population. Br J Surg 2010;97(12):1805-13.

[12] Joosse P, Schep NW, Goslings JC, Regional Trauma Network TraumaNet AMC Collaborators. Injury profiles related to mortality in patients with a low injury severity score: a case-mix issue? J Trauma 2012;73(1):179-85.
[13] Frankema SPG, Edwards MJR, Steyerberg EW, Van Vugt AB. Predicting survival after trauma: a comparison of TRISS and ASCOT in the netherlands. Eur J Trauma 2002;28(6):355-64.

[14] de Munter L, Polinder S, Lansink K, Cnossen M, Steyerberg E, de Jongh MAC Mortality prediction models in the general trauma population: a systematic review. Injury 2017;48(2):221-9.

[15] WARLTR. Landelijke traumaregistratie-toelichting uitkomstevaluatie 2015 TRISS model Nederlandse coëfficiënten en SMR. resultaten 2015 regio: Netwerk acute zorg brabant. 2016.

[16] Association for the Advancement of Automotive Medicine. Abbreviated injury scale; 1990 revision: update 98. AAAM; 1998.

[17] Gomez D, Haas B, Hemmila M, Pasquale M, Goble S, Neal M, et al. Hips can lie: impact of excluding isolated hip fractures on external benchmarking of trauma center performance. J Trauma 2010;69(5):1037-41.

[18] American College of Surgeons. National trauma data bank: NTDB research data set admission year 2008. User manual. 2010 February.

[19] Little R, Rubin D. Statistical analysis with missing data. Wiley series in probability and mathematical statistics. John Wiley \& Sons; 2014

[20] Bergeron E, Lavoie A, Belcaid A, Ratte S, Clas D. Should patients with isolated hip fractures be included in trauma registries? J Trauma 2005;58(4):793-7.

[21] Steyerberg EW. Chapter 9: coding of categorical and continuous predictors. Clinical prediction models: a practical approach to development, validation, and updating. Springer Science \& Business Media; 2010. p. 159-73.

[22] Royston P, Altman DG, Sauerbrei W. Dichotomizing continuous predictors in multiple regression: a bad idea. Stat Med 2006;25(1):127-41.

[23] Quail JM, Lix LM, Osman BA, Teare GF. Comparing comorbidity measures for predicting mortality and hospitalization in three population-based cohorts. BMC Health Serv Res 2011;11(1):1.

[24] Glance LG, Dick AW, Mukamel DB, Meredith W, Osler TM. The effect of preexisting conditions on hospital quality measurement for injured patients. Ann Surg 2010;251(4):728-34.

[25] Thompson HJ, Rivara FP, Nathens A, Wang J, Jurkovich GJ, MacKenzie EJ. Development and validation of the mortality risk for trauma comorbidity index. Ann Surg 2010;252(2):370-5.

[26] Jones JM, Skaga NO, Søvik SO, Lossius HM, Eken T. Norwegian survival prediction model in trauma: modelling effects of anatomic injury, acute physiology, age, and co-morbidity. Acta Anaesthesiol Scand 2014;58(3):30315.

[27] Bouamra O, Wrotchford A, Hollis S, Vail A, Woodford M, Lecky F. A new approach to outcome prediction in trauma: a comparison with the triss model. J Trauma 2006;61(3):701-10.

[28] Bergeron E, Rossignol M, Osler T, Clas D. Improving the TRISS methodology by restructuring age categories and adding comorbidities. J Trauma 2004;56 (4):760-7.

[29] Moon J, Seo B, Jang J, Lee J, Moon H. Evaluation of probability of survival using trauma and injury severity score method in severe neurotrauma patients. J Korean Neurosurg Soc 2013;54(1):42-6.

[30] Champion HR, Copes WS, Sacco WJ, Frey CF, Holcroft JW, Hoyt DB, et al. Improved predictions from a severity characterization of trauma (ASCOT) over trauma and injury severity score (TRISS): results of an independent evaluation. J Trauma 1996;40(1):42-9.

[31] Moore L, Lavoie A, LeSage N, Liberman M, Sampalis JS, Bergeron E, et al. Multiple imputation of the glasgow coma score. J Trauma 2005;59(3):698704.

[32] O'Reilly GM, Jolley DJ, Cameron PA, Gabbe B. Missing in action: a case study of the application of methods for dealing with missing data to trauma system benchmarking. Acad Emerg Med 2010;17(10):1122-9.

[33] Newgard CD. The validity of using multiple imputation for missing out-ofhospital data in a state trauma registry. Acad Emerg Med 2006;13(3):314-24.

[34] de Munter L, ter Bogt NC, Hesselink DD, de Jongh MA. Imputation strategies in the trauma registration. J Trauma 2017;83(5):828-36.

[35] Palmer CS, Franklyn M. Assessment of the effects and limitations of the 1998 to 2008 abbreviated injury scale map using a large population-based dataset. Scand J Trauma Resusc Emerg Med 2011;19(1):1. 